\title{
A Study of the Molecular-weight Distribution of Binder Pitches for Carbon Blocks
}

Jong Hoon Cho, Min II Kim, Ji Sun Im*

Jong Hoon Cho - C1 Gas \& Carbon Convergent Research, Korea Research Institute of Chemical Technology (KRICT), Daejeon 34114, Republic of Korea; Department of Chemical Engineering and Applied Chemistry, National University, Daejeon 341343, Republic of Korea.

E-mail address: chojh63@krict.re.kr (J.H. Cho)

Min II Kim - C1 Gas \& Carbon Convergent Research, Korea Research Institute of Chemical Technology (KRICT), Daejeon 34114, Republic of Korea;

E-mail address: mikim@krict.re.kr (M.I. Kim)

Ji Sun Im - C1 Gas \& Carbon Convergent Research, Korea Research Institute of Chemical Technology (KRICT), Daejeon 34114, Republic of Korea; Advanced Materials and Chemical Engineering, University of Science and Technology (UST), Daejeon 34113, Republic of Korea 
Phone: +82-42-860-7366, Fax: + 82-42-860-7388

E-mail address: jsim@krict.re.kr (J.S. Im) 


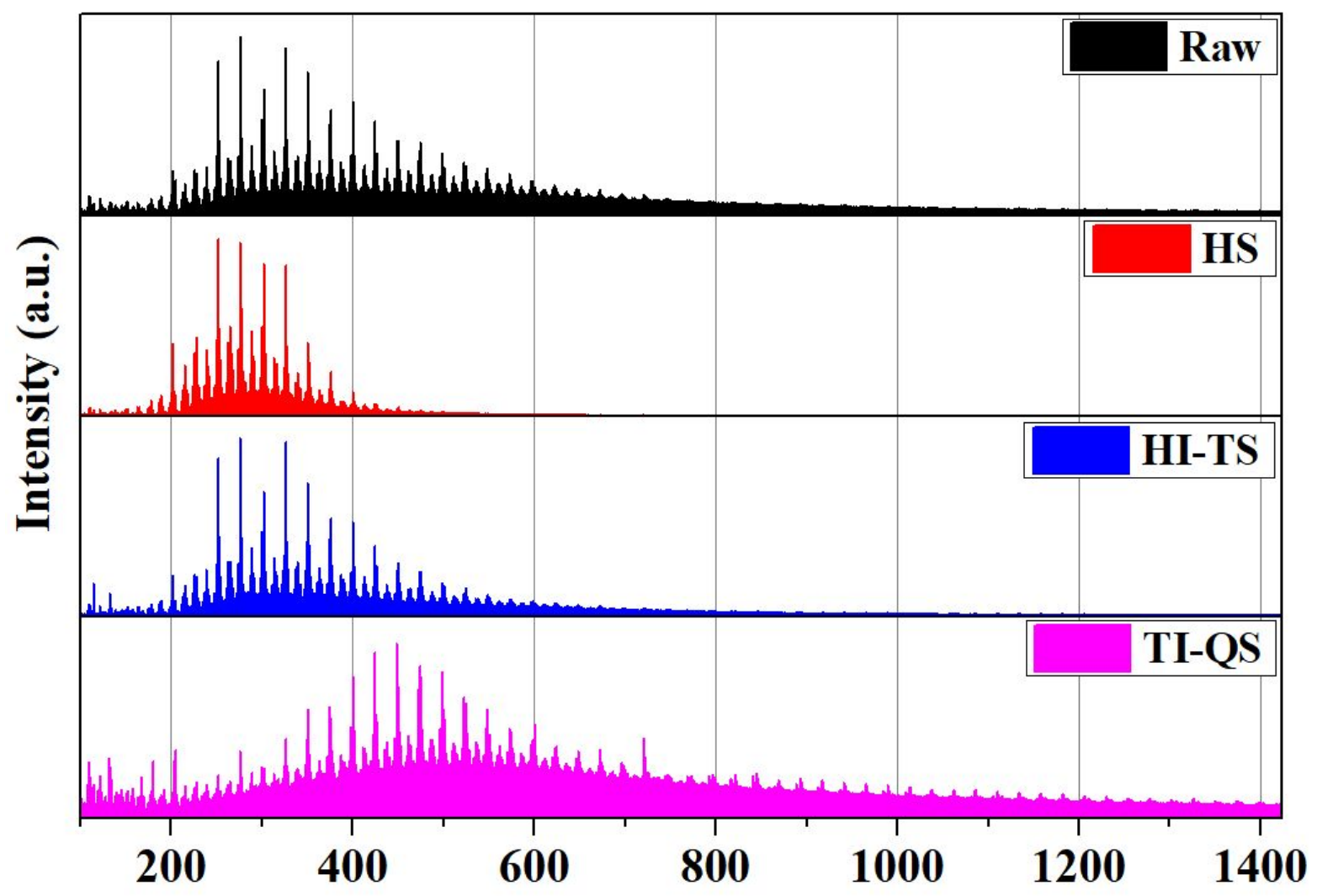

Figure S1. MALDI-TOF data of pitch fraction and raw pitch 


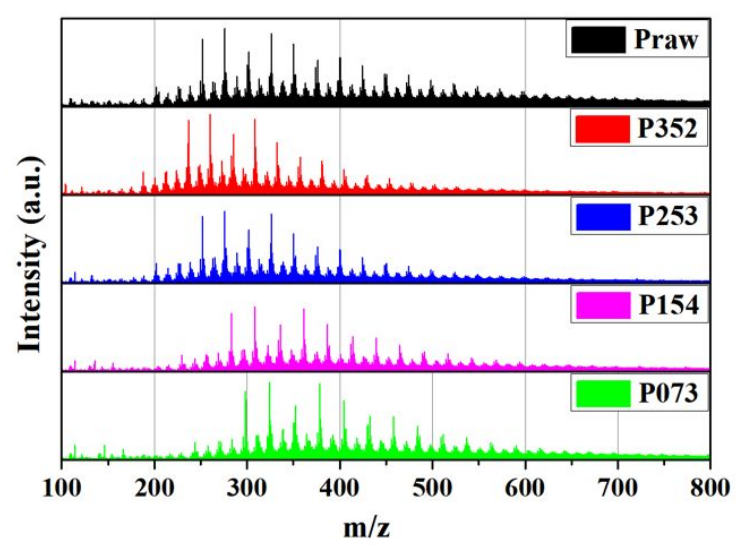

(a) MALDI-TOF

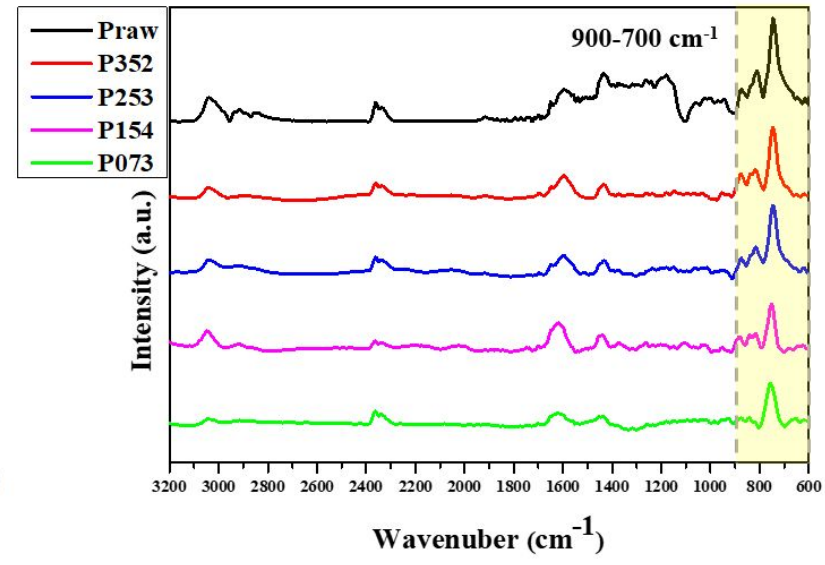

(b) FT-IR

Figure S2. FT-IR and MALDI-TOF analysis of re-fabricated pitches 
Table S1. Real density, apparent density and porosity of block according to pitch

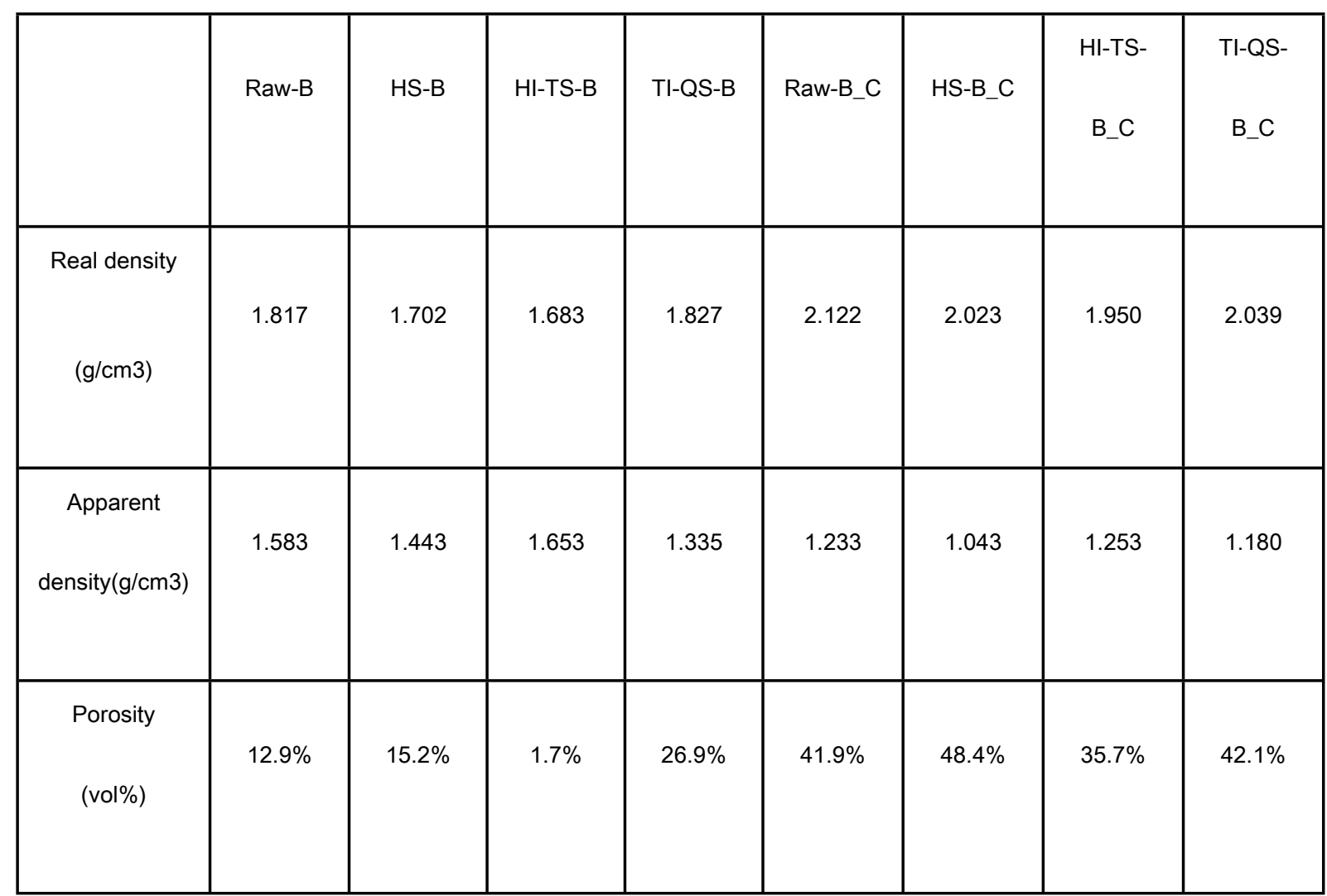

fraction

The real density and apparent density of the molded bodies were measured, as shown in Table 4. Based on these results, their porosity was approximately estimated. The real density of the green blocks made of the combined pitch fractions was found to increase with increasing molecular weight. However, both the green 
block HS-B made of HS and the green block TI-QS-B made of TI-QS exhibited low apparent density because severe cracks occurred on the surface. After the carbonization process, HI-TS showed the lowest real density. However, both HS-B and TI-QS-B showed a real density similar to Raw-B.

Meanwhile, HI-TS-B_C exhibited the lowest porosity, while HS-B_C showed the highest porosity. If the porosity of the carbon blocks is determined by the $\mathrm{CV}$ measured, it increased in the order of HS-B_C $<$ HI-TS-B_C $<$ Raw-B_C $<$ TI-QSB_C. Actually, however, HI-TS-B_C had the lowest porosity, and TI-QS-B_C showed an even higher porosity than the raw carbon block. This was because cracks were generated in TI-QS-B_C during the green block manufacturing process, and these cracks then facilitated the discharge of gases that were generated in the carbonization process, unlike other blocks with no cracks. For reference, the CV of TI-QS was $76 \mathrm{wt} \%$. This implies that about $24 \mathrm{wt} \%$ of it was converted into a gaseous form and then discharged. Therefore, it was deemed that in TI-QS_C, the gases generated from the binder pitch were not discharged, and thus closed pores were formed in smaller numbers. 